Chirurg 2019 90 (Suppl 2):S72 https://doi.org/10.1007/s00104-019-0879-3 Online publiziert: 13. Februar 2019

(c) Springer Medizin Verlag GmbH, ein Teil von Springer Nature 2019

\section{Originalpublikation}

Fretland ÅA, Dagenborg VJ, Bjørnelv GMW et al (2018) Laparoscopic versus open resection for colorectal liver metastases. Ann Surg 267:199-207

Hintergrund. Für Patienten mit einem hepatisch metastasierten kolorektalen Karzinom (KRK) stellt die Resektion der Lebermetastasen (LM) die einzige kurative Option dar. Während die laparoskopische Resektion des Primarius beim KRK mittlerweile etabliert und weit verbreitet ist, ist die Studienlage zur laparoskopischen Resektion von LM spärlich. Die OSLO-COMET-Studie vergleicht als erste randomisierte Studie die laparoskopische und die offene Leberresektion bei kolorektalen LM.

Methoden. Zwischen Februar 2012 und Januar 2016 wurden am Universitätsklinikum Oslo 280 Patienten mit resektablen LM in die monozentrische, randomisierte OSLO-COMET-Studie eingeschlossen. Primärer Endpunkt der Studie war die postoperative 30-Tage-Komplikations-Rate (Accordion-Grad 2 und höher). Zu den sekundären Endpunkten zählten unter anderem die Operationszeit, die Krankenhausverweildauer und die Rate an R1-Resektionen.

Ergebnisse. In der Studie wurde offen wie laparoskopisch ein parenchymsparendes Vorgehen angestrebt. Die perioperative Komplikationsrate betrug 31\%(44

Dieser Beitrag wurde erstpubliziert in Der Chirurg (2018) 89:240-240. https://doi.org/10. 1007/s00104-018-0612-7

M. Schrempf $\cdot$ M. Anthuber

Klinik für Allgemein-, Viszeral- und Transplantationschirurgie, Klinikum Augsburg, Augsburg, Deutschland

\title{
Laparoskopische vs. offene Resektion von kolorektalen Lebermetastasen
}

von 144) in der Gruppe mit offener Resektion im Vergleich zu 19\% (24 von 129 ) in der Gruppe mit laparoskopischer Resektion (Differenz 12\% [95\%KI 1,67-21,8; $p=0,021])$. In der laparoskopischen Gruppe erfolgte in $7 \%$ der Fälle ein Umstieg zur Laparotomie oder handassistieren Laparoskopie. Die mediane Krankenhausverweildauer war für Patienten mit offener Resektion signifikant länger als für Patienten mit laparoskopischer Resektion (96h vs. $53 \mathrm{~h}, p<0,001)$. Operationszeit, Blutverlust, 30-Tage-Wiederaufnahme-Rate und Anteil an R1-Resektionen unterschieden sich zwischen den beiden Gruppen nicht.

Diskussion und Fazit. In dieser randomisierten Studie wurde für die laparoskopische Resektion von LM beim KRK ein Vorteil in Bezug aufdie Komplikationsrate und Krankenhausverweildauer beivergleichbarer R1-Resektions-Rate nachgewiesen. Dieses Ergebnis wird durch zwei retrospektive Arbeiten gestützt $[1,3]$. Leberresektionen sind oft schwer miteinander zu vergleichen, da je nach Tumorlokalisation bei gleicher Tumorgröße ein erheblicher Unterschied im Schwierigkeitsgrad der Operation besteht. Es wurden daher verschiedene Scoring-Systeme entwickelt, die eine bessere Vergleichbarkeit von Leberresektionen ermöglichen sollen. In der vorliegenden Arbeit unterschieden sich die beiden Gruppen hinsichtlich des medianen Modified Liver Surgery Complexitiy Score [2] nicht. Der Anteil an Patienten mit vorausgegangener Leberresektion war in der Gruppe mit laparoskopischer Resektion sogar höher als in der Gruppe mit offener Resekti- on. $\mathrm{Zu}$ beachten ist, dass diese Ergebnisse aus einem spezialisierten Zentrum mit großer Erfahrung in der laparoskopischen Leberchirurgie stammen und vermutlich nicht ohne weiteres flächendeckend übertragbar sind.

\section{Korrespondenzadresse}

\section{Prof. Dr. M. Anthuber}

Klinik für Allgemein-, Viszeral- und

Transplantationschirurgie, Klinikum Augsburg Stenglinstr. 2, 86156 Augsburg, Deutschland matthias.anthuber@klinikum-augsburg.de

Interessenkonflikt. M. Schrempf und M. Anthuber geben an, dass kein Interessenkonflikt besteht.

\section{Literatur}

1. Cipriani F, Rawashdeh M, Stanton L et al (2016) Propensity score-based analysis of outcomes of laparoscopic versus open liver resection for colorectal metastases. Br J Surg 103:1504-1512

2. Lee MK, Gao F, Strasberg SM (2016) Completion of a liver surgery complexity score and classification based on an international survey of experts. J Am Coll Surg 223:332-342

3. Lewin JW, O'Rourke NA, Chiow AKH et al (2016) Long-term survival in laparoscopic vs open resection for colorectal liver metastases: inverse probability of treatment weighting using propensity scores. HPB (Oxford) 18:183-191 